Check for updates

Cite this: Mater. Chem. Front. 2020, 4, 1089

Received 31st December 2019 Accepted 29th January 2020

DOI: 10.1039/c9qm00788a

rsc.li/frontiers-materials

\section{Recent advances on polydiacetylene-based smart materials for biomedical applications}

\begin{abstract}
Fang Fang, ${ }^{a}$ Fanling Meng (D) a and Liang Luo (D) *abc
Polydiacetylene (PDA) is a kind of highly conjugated polymer with unique colorimetric and optical properties. A blue and non-fluorescent PDA can be formed upon $254 \mathrm{~nm}$ UV irradiation or $\gamma$-ray irradiation via 1,4-polymerization of diacetylene monomers, which will transition to red and fluorescent PDA when exposed to environment stimuli, such as $\mathrm{pH}$, temperature, electric and mechanical stresses, and ligand-receptor interactions. As a result, PDA-based materials are particularly attractive for biosensing applications. In addition, the rigid conjugated backbone of PDA derived from its unique topochemical polymerization method also endows exceptional mechanical strength and stability to PDA-based biomaterials, such as liposomes, micelles, and bioscaffolds. The versatility of PDA-based smart materials enables their application in a wide range of biomedical areas. In this review, we briefly summarized the recent progress of biomedical applications of PDA as smart materials in biosensors, drug delivery and bioimaging, as well as tissue engineering. The challenges and outlooks of PDA-based smart materials were also discussed.
\end{abstract}

${ }^{a}$ National Engineering Research Center for Nanomedicine, College of Life Science and Technology, Huazhong University of Science and Technology, Wuhan, 430074, China.E-mail: liangluo@hust.edu.cn

${ }^{b}$ Hubei Key Laboratory of Bioinorganic Chemistry and Materia Medica, School of Chemistry and Chemical Engineering, Huazhong University of Science and Technology, Wuhan 430074, China

${ }^{c}$ Shenzhen Huazhong University of Science and Technology Research Institute, Shenzhen 518057, China

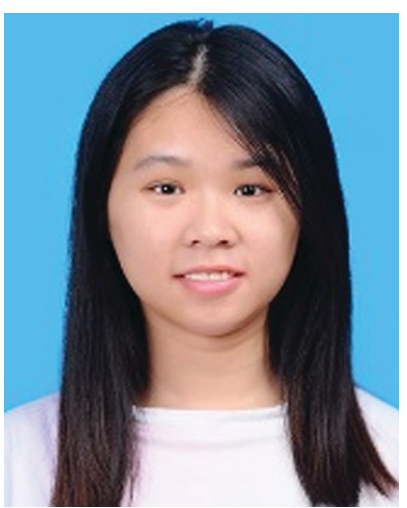

Fang Fang
Fang Fang is currently an undergraduate student at College of Life Science and Technology in Huazhong University of Science and Technology. Her research interest focuses on the design and synthesis of novel polydiacetylenebased smart materials for cancer diagnosis and treatment, under the supervision of Professor Liang Luo.

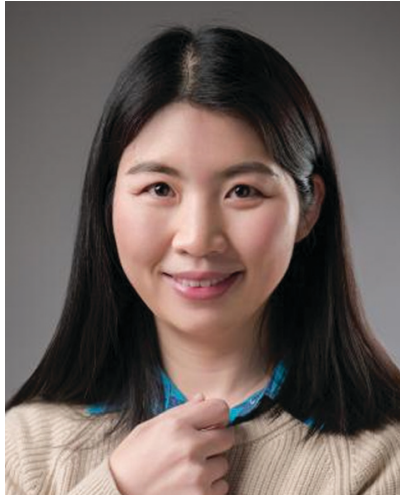

Fanling Meng
Fanling Meng received her bachelor's degree at Tianjin University of Science and Technology and master's degree at Nankai University. She received her PhD at State University of New York at Stony Brook, working with Daniel P. Raleigh. Following postdoctoral research work with Peter Tessier at Rensselaer Polytechnic Institute, she came to Huazhong University of Science and Technology in 2016, with a research focus on amyloid inhibition and peptide self-assembly. 
A
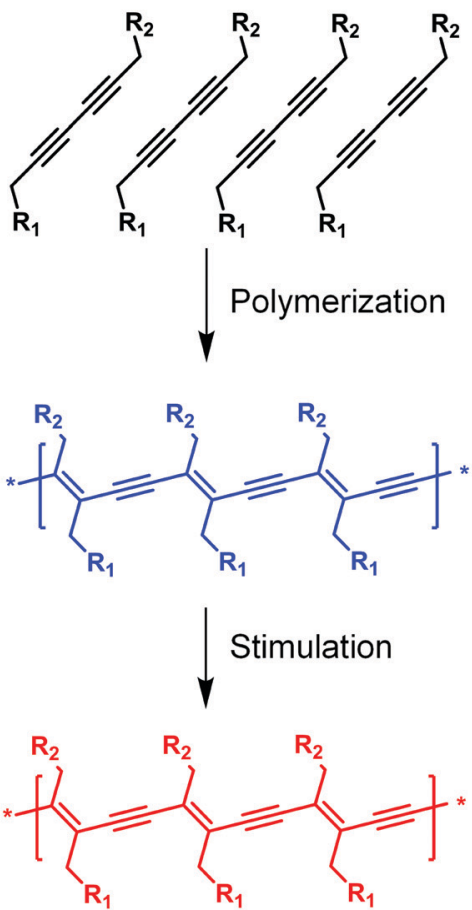
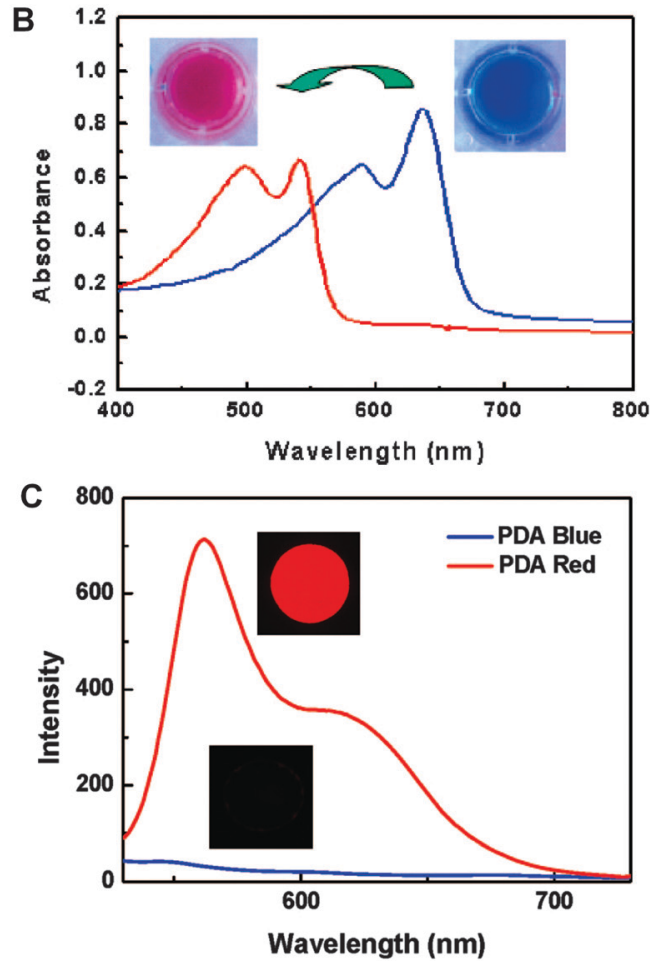

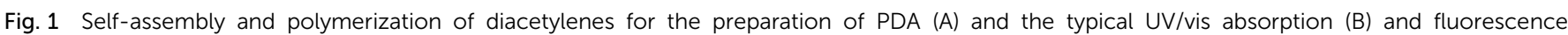

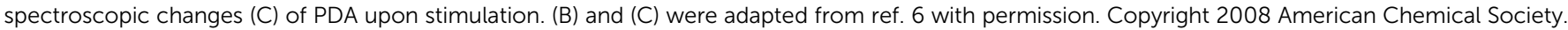

distinctive feature of PDA is its preparation method, i.e., topochemical polymerization in the solid state, ${ }^{7,12}$ which requires ordered alignment of diacetylene monomers. PDA can be formed upon $254 \mathrm{~nm}$ UV irradiation or $\gamma$-irradiation via 1,4-addition polymerization of aligned diacetylene monomers with specific packing geometric parameters (Fig. 1). The self-assembly of the monomers driven by the noncovalent interaction of the monomer side chains, such as hydrophobic effect, van der Waals'

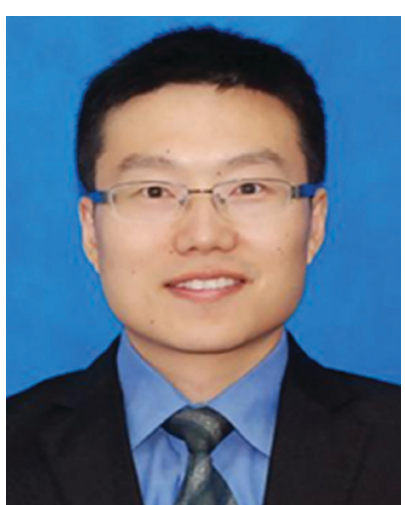

Liang Luo
Liang Luo earned his BS and MS degrees in Chemistry in Nankai University, and obtained his PhD in Chemistry from State University of New York at Stony Brook in 2009 under the supervision of Prof. Nancy S. Goroff. After completing postdoctoral research at Department of Chemical Engineering and Materials Science in University of Minnesota with Prof. C. Daniel Frisbie, he joined Bristol-Myers Squibb Company as a Research Investigator. $\mathrm{He}$ is currently a Professor in the College of Life Science and Technology at Huazhong University of Science and Technology. His research focuses on exploring novel conjugated organic materials for biomedical applications. interactions, and hydrogen bonding, offers a great opportunity for the appropriate alignment of the monomers. ${ }^{13-15}$ The topological polymerization process occurs without initiator or catalyst, which ensures the production of PDAs with extremely high planarity and uniformity. ${ }^{4}$

The freshly prepared PDAs appear blue in color owing to the reduced $\pi-\pi^{*}$ bandgap by the quasi-one-dimensional ene-yne conjugated polymer backbones. ${ }^{16,17}$ Interestingly, when the blue PDAs are exposed to environmental stimuli, such as $\mathrm{pH}$ values, temperature, electrical stress, mechanical stress, and ligand-receptor interactions, they usually undergo a visible blue-to-red color change associated with a clear hypsochromic shift in the absorption spectrum. ${ }^{8,18-23}$ In addition, PDAs are typically fluorescent in their red phase, since the lowest excited state has $B_{u}$ symmetry that causes the radiation decay and consequently produce strong fluorescence peaked at around $650 \mathrm{~nm} \cdot{ }^{14,24}$ As a comparison, the blue-phase PDAs usually exhibit very weak fluorescence, because the lowest excited state is $A_{g}$ symmetry, a dipole-forbidden transition. ${ }^{25}$ In most cases, the blue-to-red phase transition is irreversible, and the fact that these optical changes can be observed directly by naked eyes or by UV/vis absorption and fluorescence spectroscopy make PDAs excellent candidates of biosensors.

The properties and applications of PDA materials have been reviewed and discussed over the years. ${ }^{4,6-9,26,27}$ Previous studies on the application of PDA materials in the forms of liposomes, vesicles, Langmuir-Blodgett films, and strips have been reported as well. ${ }^{28-38}$ With the continuous discovery of more intriguing features of PDA, the application field of PDA has also been broadened. 
In particular, the rigid conjugated backbone provides exceptional mechanical properties and good biocompatibility to PDA-based supramolecular architectures, making researchers start to exploit their biomedical applications beyond biosensors, such as drug delivery, bioimaging, and tissue engineering. Herein, we summarized recent advances of PDA-based smart materials for biomedical applications in this review, with particular emphasis on the designing strategy of PDA-based biosensors, as well as the applications of PDAs in drug delivery and tissue engineering that are less focused in previous reviews. This review should provide new insights into further exploiting PDA-based biomaterials for future biomedical applications.

\section{Biological sensing}

\subsection{Directly detecting biological targets}

The direct detection method is most commonly used in designing PDA-based biosensors, attributed to the easy functionalization of PDA as well as the specificity of modified PDA sensors. When linking the head groups of PDA to a ligand that can interact with biomolecules specifically, or conjugating antibodies onto the PDA assemblies, PDA can be directly used as sensors with specific responses to DNA, virus, bacteria, glucose, and proteins. There have been many PDA-based biosensors developed recently based on this method. ${ }^{39-44}$

In 2009, Sim et al. reported a cross-linked PDA-based multiplex biosensor platform. ${ }^{45}$ PDA liposomes were cross-linked by ethylenediamine and immobilized on a solid substrate. After being functionalized by the anti-pathogen antibodies to the head of group of PDA liposomes, the cross-linked and immobilized PDA-based biosensor chips exhibited approximately 10 times higher detection sensitivity than the ones without crosslinking. The PDA liposomes-based multiplex biosensor chips with great specificity was able to perform both quantitative and qualitative analyses of target pathogens without the pretreatment of samples, enabling a facile and simple detection of pathogens. Later, Jung and Park developed a 9-aminoacridine (9AA) functionalized PDA liposomes that exhibited great specificity in clinical double-stranded DNA (dsDNA) samples detection. ${ }^{46}$ The planar aromatic dye 9AA was able to insert orthogonally into dsDNA via dispersal forces and electrostatic interactions, and induced stress-mediated morphological and conformational change of PDA liposomes and a consequent blue-to-red chromism. ${ }^{47}$ This sensor was used for dsDNA detection with a detection limit of $20 \mathrm{nM}$ in $1 \mathrm{~h}$.

In fact, PDA-based materials are promising sensors to detect biomolecules in living cells owing to their good affinity to biological targets after appropriate functionalization. Inspired by the pioneering efforts in developing methods for the in situ detection of cell surface sialic acid (SA), ${ }^{48-50}$ Wang et al. reported a fluorescence turn-on PDA liposome sensor with phenylboronic acid (PBA) tags for SA detection and cell-surface glycan in situ imaging. ${ }^{51}$ Since PBA was demonstrated to have a stronger binding with SA than with other saccharides at physiological $\mathrm{pH}$ conditions, ${ }^{48}$ the PDA liposomes-based sensor containing PBA ligands could recognize the SA-terminated glycans on living cell surfaces. Another work reported in 2019 by Lim et al. presented a PDA-based paper sensor to detect highly infectious pH1N1 virus. ${ }^{38}$ By conjugating with the antibodies that can specifically bind to influenza A virus nucleoprotein, the PDA-immobilized polyvinylidence fluoride membrane enabled qualitative examination of pH1N1 virus with the naked eyes (Fig. 2A). On the basis of this virus-responsive chromism, the authors developed a smartphonebased point-of-care testing device, so that low concentration of virus can be read directly on a smartphone app.

\subsection{Indirect detection of biological targets}

Unlike the direct detection method that needs laborious pre-synthesis of a functionalized diacetylene monomer or functionalization of PDA side chains, indirect detection method utilizes unmodified PDA vesicles or liposomes to detect the products of enzyme's hydrolyzation or the molecules secreted by cells. Since PDA itself typically undergoes blue-tored chromatic transition in response to environment stimuli, the indirect detection method can detect targets by responding to the environmental change triggered by the targets, offering a good sensitivity in the case where it is difficult to find suitable binding receptors of the targets.

In 2016, Park et al. successfully fabricated a label-free bacteria PDA sensor with high selectivity. ${ }^{52}$ Instead of designing receptors that were specific to bacteria, they focused on the interaction between the chemicals released from bacteria and the PDA liposomes, and finally realized indirect detection of surfactin-producing bacteria. By comparing the color changes of Luria-Bertani-agars-incorporated PDA in the presence of surfactinless Gram-positive bacterium (Bacillus subtilis SSB466), surfactinproducing Gram-positive bacterium (Bacillus subtilis NCIB3610), and Gram-negative bacterium (Pseudomonas aeruginosa 14), they found that although growing less vigorously than the other two strains of bacteria, the NCIB 3610 resulted in more visible color change of the PDA-containing agars, demonstrating a detection mediated by PDA and bacteria-derived chemicals.

Very recently, Kim et al. developed a sensitive, inexpensive, and label-free urease sensor based on the colorimetric response of PDA vesicles to $\mathrm{pH}$ change. ${ }^{36}$ The blue-to-red colorimetric transition of carboxyl-terminated PDA vesicles was induced by the deprotonation of the carboxyl head groups by ammonia formed during urea hydrolyzation. They also used this method to screen the urease inhibitor acetohydroxamic acid, and the half-maximal inhibition value $\left(\mathrm{IC}_{50}\right)$ was close to previous research $(50 \mathrm{mM}) .{ }^{53} \mathrm{Xu}$ et al. reported a simple colorimetric assay for indirect detection of alkaline phosphatase (ALP) activity based on PDA liposomes and competitive binding between pyrophosphate (PPi) and $\mathrm{Ca}^{2+}$ (Fig. 3A). ${ }^{54}$ The unmodified 10,12-pentacosadiynoic acid (PCDA) liposomes exhibited a blue-to-red color change when binding with $\mathrm{Ca}^{2+}$. However, $\mathrm{PPi}$, a natural substrate for ALP ${ }^{55}$ could effectively block the binding between PCDA and $\mathrm{Ca}^{2+}$ due to its high chelation affinity with $\mathrm{Ca}^{2+}$ in aqueous solutions (Fig. 3B). After adding ALP to the mixed system, PPi was hydrolyzed and no longer had a strong interaction with $\mathrm{Ca}^{2+}$, therefore resuming the 


\section{A}

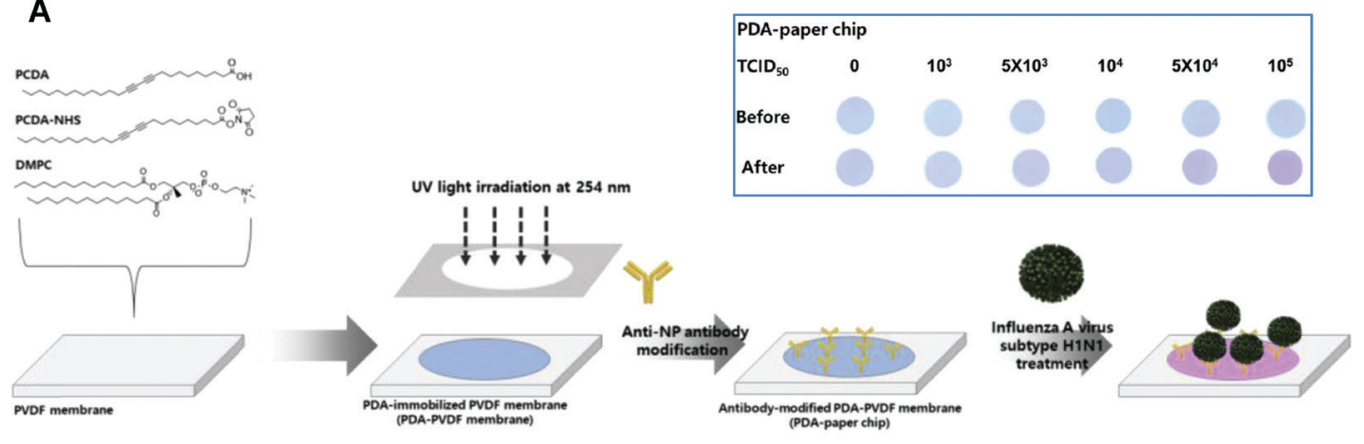

B
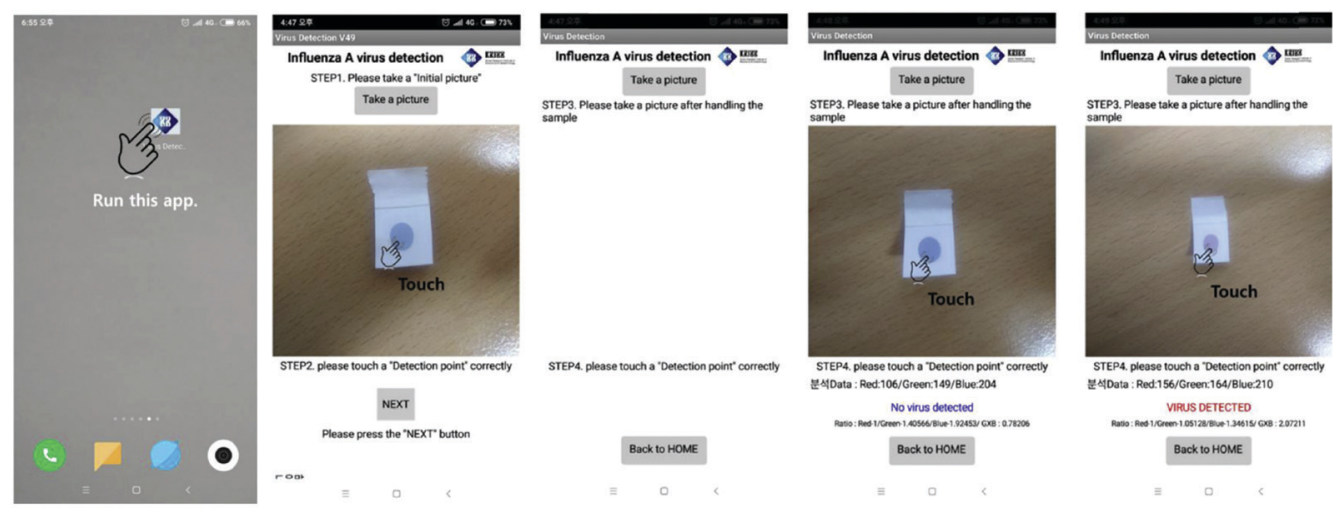

Fig. 2 (A) Preparation of PDA-paper chip. Inset: Color transition images of a PDA-paper chip being treated with different concentrations of pH1N1 virus for $3 \mathrm{~h}$ at room temperature, respectively. (B) Smartphone-based virus sensing procedure. Adapted from ref. 38 with permission. Copyright 2019 Elsevier B.V.

colorimetric response of PDA to $\mathrm{Ca}^{2+}$. Compared with the aforementioned direct detection method, the marks of the indirect detection method of PDA-based smart materials include easy preparation, cost-effectiveness, and more importantly, a reversible label-free feature that is particularly suitable for reusable devices.

\subsection{Sandwich type detection method}

Similar to enzyme linked immunosorbent assay (ELISA), some PDA-based sensing systems also employ a sandwich method to enhance the fluorescent signal and sensitivity. By introducing an external stimuli receptor on the targets bound to PDA, such as magnetic beads, antibodies, and enzymes, the sandwich-type PDA biosensors possess the capability to detect targets at ultralow concentrations with great sensitivity and selectivity.

In 2010, Sim et al. reported a strategy to amplify fluorescent signals and improve detection sensitivity, by introducing a hybrid stimulus, in the detection of prostate-specific antigen$\alpha 1$-antichymotrypsin (PSA-ACT) complex. ${ }^{56}$ The sandwich type PDA vesicle chip utilized the antibody-antigen immune reaction as the primary response and the mechanical pressure occurred by antibody-conjugated magnetic beads as the secondary response. The PDA vesicle chip exhibited a minimum detection concentration of $0.1 \mathrm{ng} \mathrm{mL}^{-1}$ for PSA-ACT complex. Later, they developed a more sensitive PDA liposome biosensor for the detection of human immunoglobulin E (hIgE). ${ }^{57}$ Instead of directly using antibody as the hIgE receptor, they immobilized an anti-hIgE aptamer on the PDA liposome biosensors. The prepared PDA liposome biosensors could detect hIgE at $1.0 \mathrm{ng} \mathrm{mL}^{-1}$ by the aptamer-based primary response and introducing polyclonal hIgE antibody as the secondary response.

In 2014, Sim and coworkers developed a micro-arrayed PDA immunosensor that could detect hIgE with ultrahigh sensitivity. ${ }^{58}$ They applied multiple stimuli to a PDA-based immunosensor chip, allowing for multistep signal enhancement of the target protein. In addition to the primary antigen-antibody immune reaction, the fluorescence signal was also boosted by the mechanical force from enzyme-catalyzed precipitation (Fig. 4). As a result, hIgE could be detected with a concentration of as low as $0.01 \mathrm{ng} \mathrm{mL} \mathrm{m}^{-1}$, which is 1000 -fold lower than by the primary immunoresponse. The above mentioned sandwich type methods enabled ultra-sensitive detection of targets, which opened up new opportunities for ultra-low concentration biomolecules such as cancer-associated proteins.

\subsection{Competitive binding method}

Different from the strategies introduced above, competitive binding method works in a way of detecting the recovery of the initial signals of PDA. This method can be used for screening target inhibitors or receptors with great specificity. The key of this method is to determine the substrate molecules or ions that not only interact with PDA to induce the blue-to-red colorimetric transition, but also possess high affinity towards target inhibitors or receptors. The competitive binding between PDA and inhibitors 
A
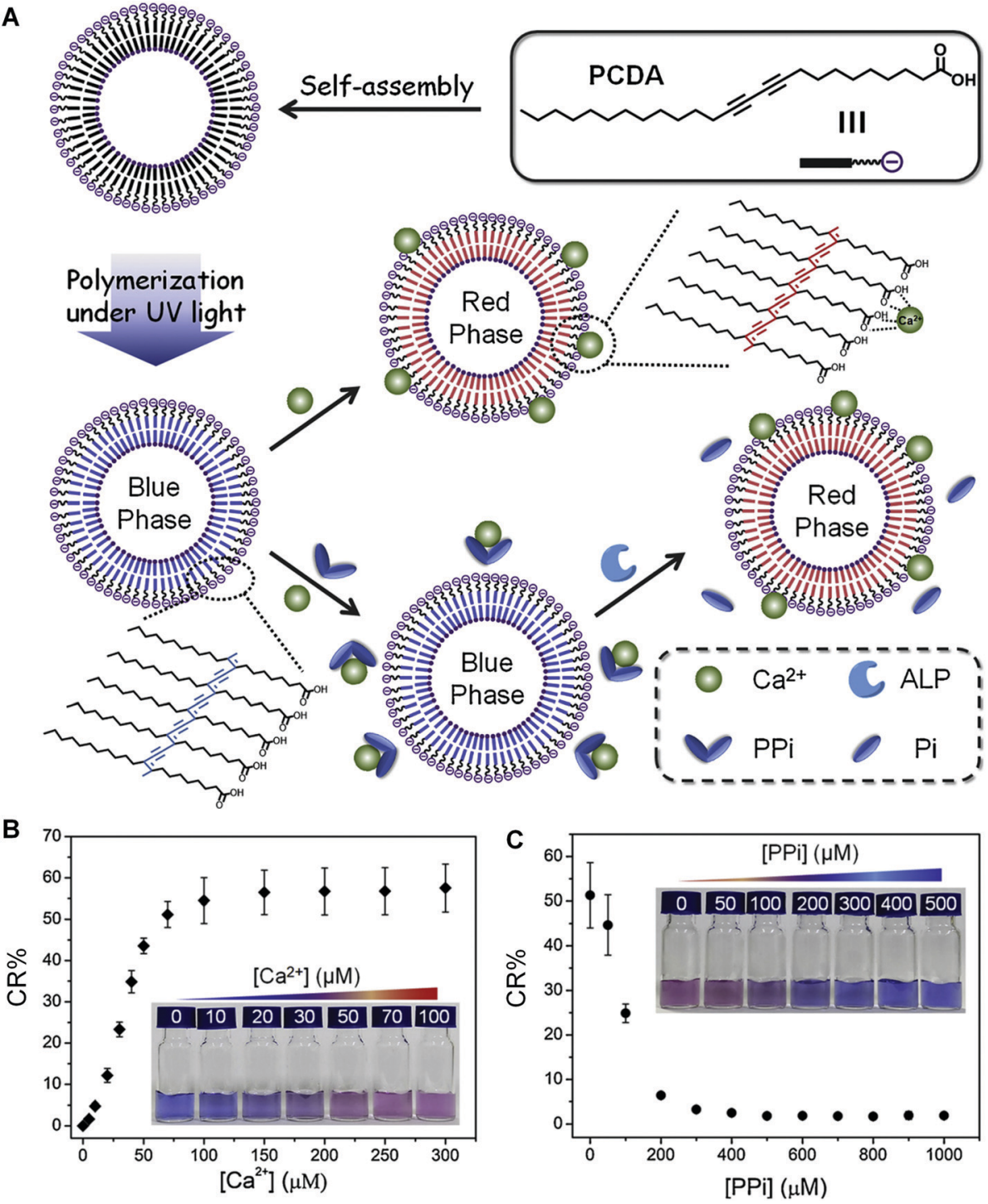

Fig. 3 (A) Schematic illustration of the preparation of PDA liposomes from pure PCDA and the principle of PDA liposomes for colorimetric sensing of ALP. (B) Corresponding CR (\%) values of PDA liposomes $(100 \mu \mathrm{M})$ in the presence of different concentrations of Ca ${ }^{2+}$. Inset: Photographs of corresponding samples. (C) Corresponding CR (\%) values of PDA liposomes $(100 \mu \mathrm{M})$ containing $\mathrm{Ca}^{2+}(100 \mu \mathrm{M})$ in the presence of different concentrations of PPi. Inset: Photographs of corresponding samples. Adapted from ref. 54 with permission. Copyright 2019 Elsevier B.V.

or receptors with the substrate molecules or ions is the basis of the method for detection.

In 2015, Brockgreitens developed a PDA liposomes-based sensor to analyze the reaction kinetics of $\alpha$-cyclodextrin $(\alpha-\mathrm{CD})$ and optically inactive surrogates, ${ }^{59}$ by analyzing the degree to which the blue-to-red color change of PDA liposome was suppressed by these reactions. A gold-nanorod functionalized PDA microtube (Au@PDA) was reported by Zou et al. in 2016 for the selective and sensitive detection of miRNA-21 (Fig. 5). ${ }^{60}$ In Au@PDA, the excitation and emission peaks were well separated, which could effectively reduce the background fluorescence interference. The fluorescence of Au@PDA was quenched when the Au@PDA microtube was initially prepared. The gold nanorod was close enough to the surface of the PDA microtube to trigger a fluorescence resonance energy transfer (FRET).
After adding miRNA-21, it could displace the complementary DNA within the surface of the microtube. As a result, the gold nanorods was removed from the PDA microtube surface, and recover the waveguide coupled tip emission of PDA microtube (Fig. 5B and C). The examples above showed that the competitive binding method has advantages of great specificity and excellent sensitivity, making it a promising candidate for the development of clinical diagnosis in complex biological environment.

\section{PDAs for drug delivery and bioimaging}

\subsection{PDA-based liposomes}

Liposomes have attracted great interests as drug carriers due to their good biocompatibility and tunability of physical 
A
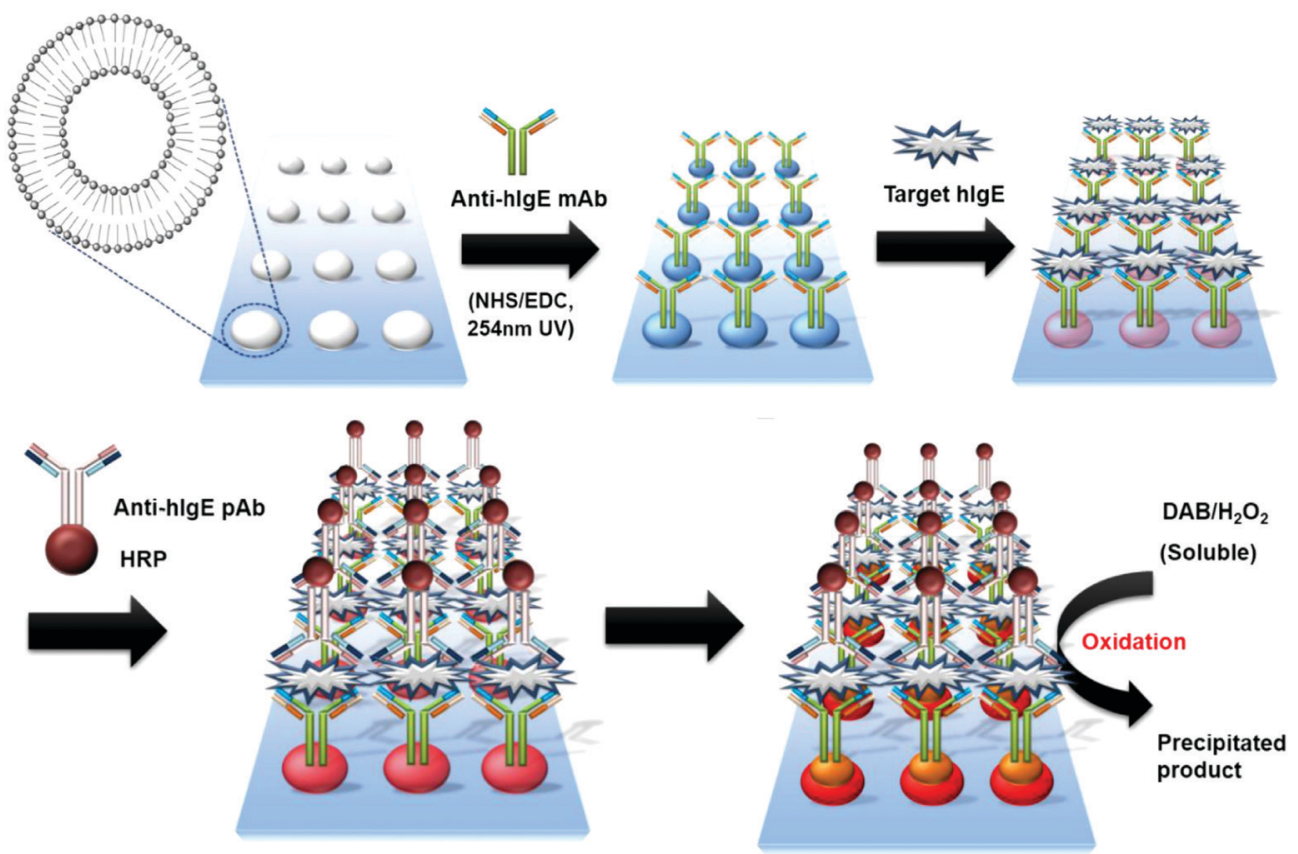

B
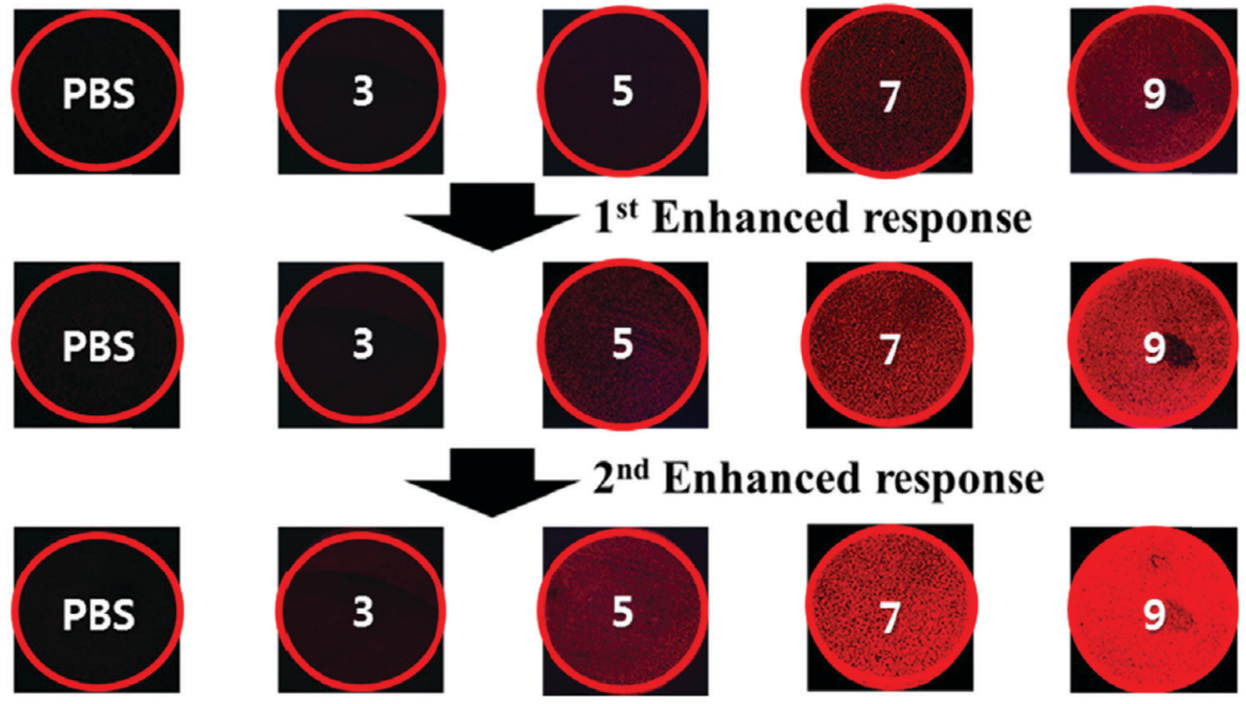

Fig. 4 (A) Schematic illustration of the preparation of the PDA liposomes-based immunosensor for the detection of target hlgE. (B) Fluorescent microscopy images and diagram representing the fluorescent signal in the detection target hlgE by the liposome immunosensor after (i) primary immune response and (ii) first enhanced response using anti-hlgE pAb-HRP. (iii) Second enhanced response resulting from enzyme catalyzed precipitate formation. Adapted from ref. 58 with permission. Copyright 2014 Elsevier B.V.

and chemical properties. The chemotherapy drugs carried in liposomes are generally cytotoxic, and the precise release of drugs at target tissues is pivotal. However, the instability of liposomes in the physiologic environment usually results in unwanted drug leakage at the non-target sites. ${ }^{61}$ PDA-based liposomes can perfectly overcome the instability issues, attributed to the rigid bilayer structures given by their conjugated backbones. What's more, PDA-based liposomes own unique features including colorimetric transition and optical properties, in addition to the major advantages of liposomes such as improved bioavailability and limited toxicity. ${ }^{62}$

In 2011, Li et al. fabricated a kind of partially polymerized liposomes composed of PDA and saturated lipids, for enhanced stability as well as precisely controlled drug release. ${ }^{63}$ The partially polymerized liposomes were prepared from the mixture of polymerizable lipids with diacetylene moieties and nonpolymerizable lipids, followed by the attachment of gold nanoparticles on the outer surface through Au-S bonds. ${ }^{64}$ The stable liposomal system could retain the encapsulated drug at $40{ }^{\circ} \mathrm{C}$ for three days. However, upon irradiation by a laser matching the surface plasmon resonance of the GNPs, the liposomal system could instantly release over $70 \%$ of its payload.

The stability and controlled release of PDA-based liposomal systems allows for a prolonged circulation time, which is particularly important for their accumulation in tumor tissues 


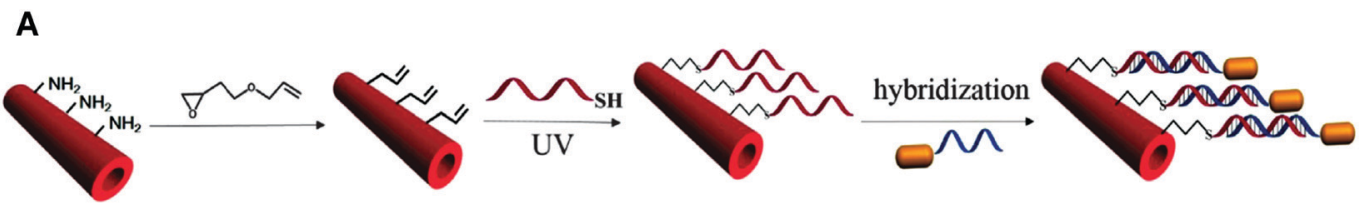

B
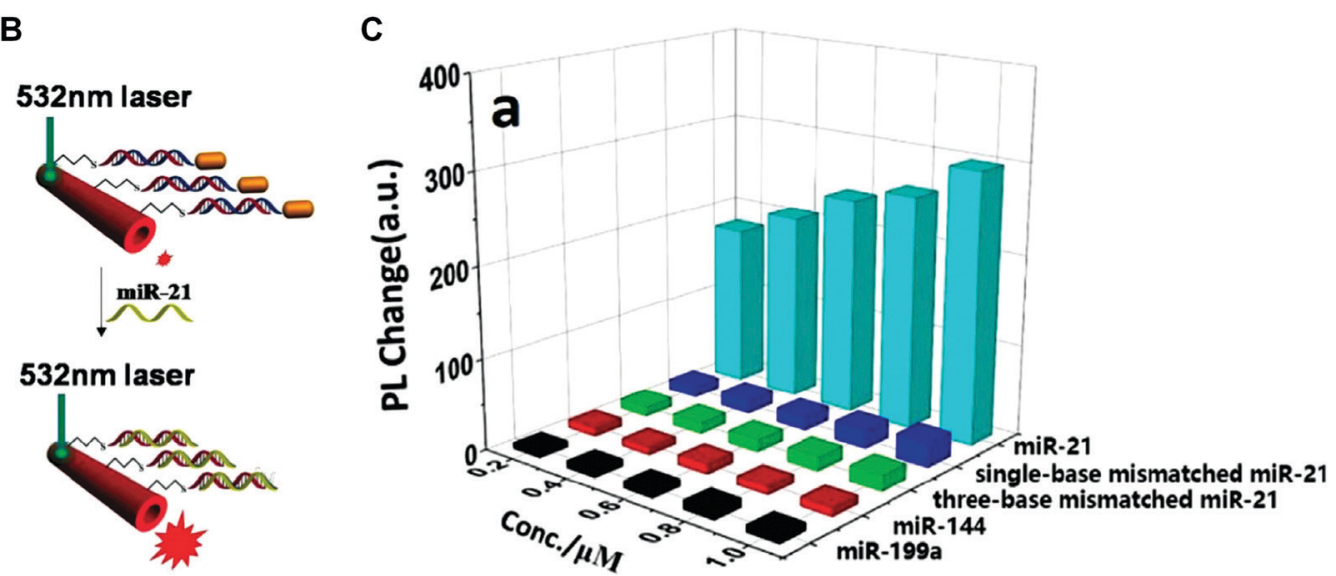

Fig. 5 (A) Schematic illustration of the Au@PDA microtubes preparation process. (B) Schematic illustration of the single Au@PDA microtube wave guide system for miRNA-21 detection. (C) Corresponding out-coupled emission change at the tip of PDA microtube upon addition of miRNA-21 or other analytes. Reproduced with permission from ref. 60. Copyright 2016 Elsevier B.V.

by the enhanced permeability and retention (EPR) effect. ${ }^{65,66}$ Abrams et al. reported the synthesis of antigen-conjugated PDA-based liposomes for vaccine development in $2017 .{ }^{67}$ The liposomes were composed of PCDA, 1,2-dimyristoyl-sn-glycero3-phosphocholine (DMPC), and PCDA-conjugated $N$-hydroxysuccinimide (PCDA-NHS), and could maintain the shape and structure in physiological conditions for a long time without fusing with cell membranes, because of the rigid ene-yne conjugation backbone structure. In their study, the immunogenicity of antigen-conjugated PDA liposomes was demonstrated, which could induce strong antibody titers in immunized animals without the use of adjuvants.

However, in the case of low vascular permeability cancers, an active targeting group that can selectively recognize specific cells is still necessary. ${ }^{68}$ An and coworkers provided a good example by developing a multifunctional folate-PDA-liposomes (FP-PLs). ${ }^{69}$ The PDA-based liposomes using folic acid derivatives as the targeting ligand enabled both tumor-targeted drug delivery and fluorescence detection (Fig. 6A). As shown in Fig. 6B, FP-PLs exhibited a significantly enhanced cellular uptake than non-functionalized PDA liposomes (P-PLs) by folate receptor-overexpressing cells via the folate receptormediated endocytosis. The docetaxel (DTX)-loading liposomes exhibited strong cytotoxicity towards Bcap-37 breast cancer cells while FP-PL itself had almost no effect on cell viability, demonstrating the potential of FP-PLs as a tumor-targeting carrier of therapeutic and imaging agents.

The above summarized examples indicated that the PDAbased liposomes are efficient in delivering polar drugs to target tissues due to their central hollow cavity as well as accumulating at target tissues via EPR effect or active targeting. In addition, their enhanced stability endowed by the rigid backbone also allows for a long circulation time that is important to the faith of nanomedicine in vivo.

\subsection{PDA based micelles}

Micelles, colloidal supramolecular nanostructures formed by self-assembly of amphiphilic molecules, are good nanocarrier candidates for hydrophobic molecules due to their amphiphilic core-shell structures. However, the micelles are dynamically unstable, so that they are very sensitive to concentrations. When diluted below the critical micelle concentration (CMC), the micelles turn to dispersed amphiphilic molecules. Therefore, the in vivo application of micelles is limited in general even though some micelle-based drugs are already on the market or in clinical trials. Among the attempts to tackle this issue, ${ }^{70,71}$ micelles made of amphiphilic molecules incorporating photoresponsive diacetylene groups represents a promising solution. Upon UV irradiation at $254 \mathrm{~nm}$, the PDA-based micelles link the amphiphilic molecules together through a polymerization step, which greatly stabilizes the structure of the micelles.

Similar to the liposomes reviewed above, micelles can also accumulate in tumor sites through EPR effect or active targeting by the functional ligands modified on micelles. To improve the tumor-targeting ability of PDA micelles, researchers had developed many different methods. For example, Doris and coworkers reported the preparation of biotin-functionalized PDA micelles which could selectively target MCF-7 cancerous cells. ${ }^{72}$ In this study, the authors compared the biotin-functionalized PDA micelles and "naked" micelles in targeting MCF-7 cells, and found that the functionalized micelles were more readily internalized. More recently, the same group prepared PDA micelles 
A

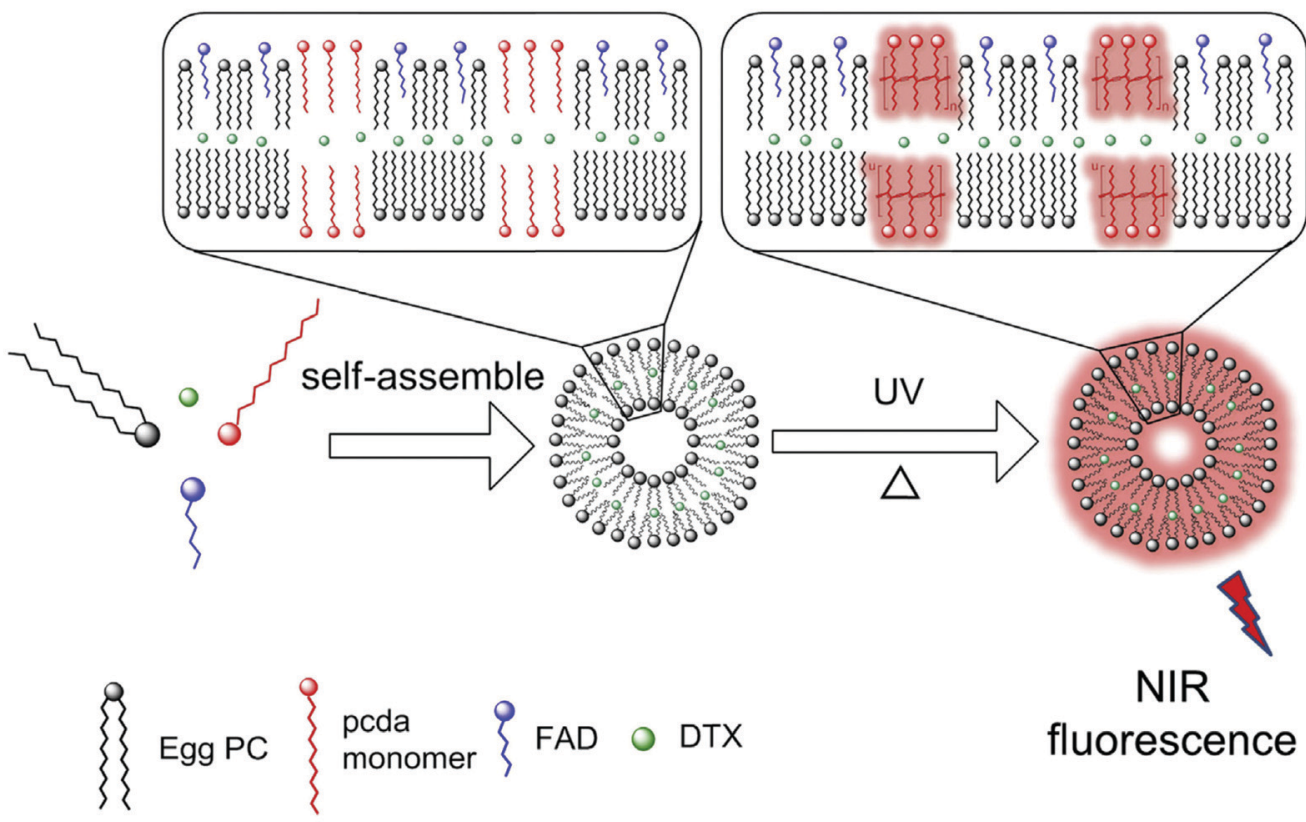

B
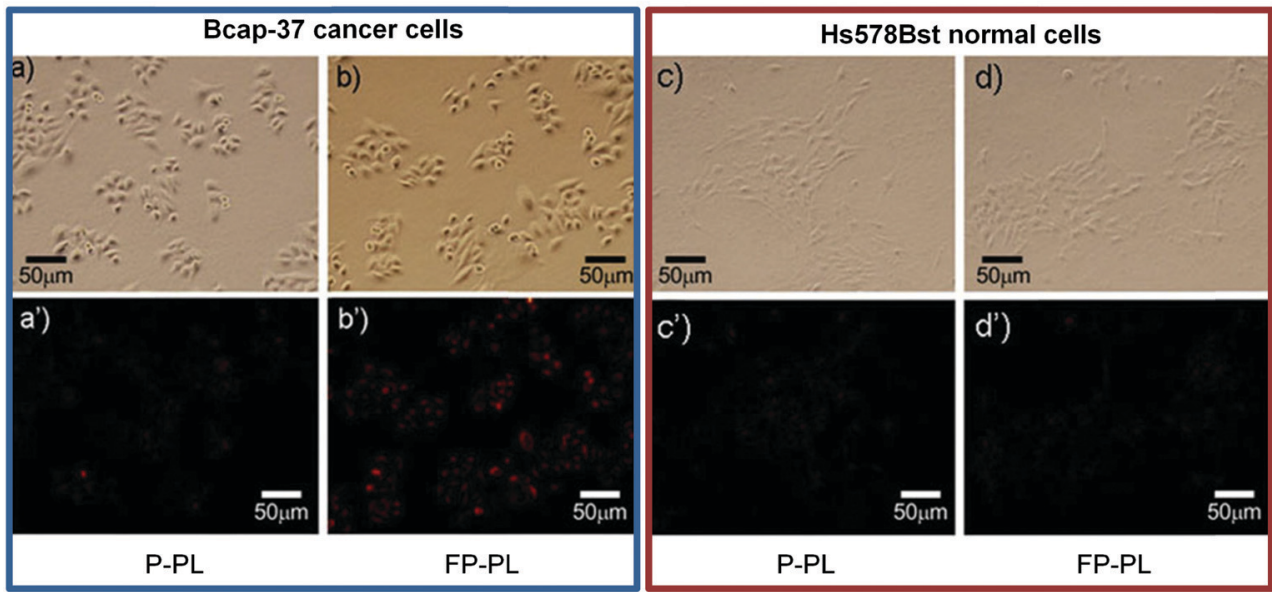

Fig. 6 (A) Schematic illustration of the preparation of FP-PL. (B) Fluorescence microscopic images of Bcap-37 cancer cells and Hs578Bst normal cells incubated with P-PLs or FP-PLs. Adapted from ref. 69 with permission. Copyright 2015 Elsevier B.V.

functionalized with a MCF-7 cells-targeting aptamer ligand by a "click and hybridization" strategy. ${ }^{73}$ Decorated with an aptamer ligand that could target Annexin A2 efficiently, the prepared PDA micelles exhibited increased MCF-7 cellular uptake, around 3 folds higher than those functionalized with the non-targeting aptamer control.

Khiar et al. coated mannose on PDA-based nanomicelles, and applied them to deliver hydrophobic drugs, taking the advantage of the tissue-specific carbohydrate-lectin interactions. $^{74}$ In this research, the obtained PDA micelles with hydrophobic inner core and hydrophilic shell were suitable for cytotoxic hydrophobic drug delivery. The bio-detectable mannose moiety capped on the PDA micelles endowed the nanovectors a high affinity towards mannose-specific lectin Concanavalin A, making them promising synthetic multivalent systems for treating pathologies mediated by carbohydratelectin interactions.
Although these functionalized PDA micelles exhibited high affinity towards target receptors in vitro, maintaining their specificity to receptors in complicated physiological environments remains a great challenge. In 2011, Mackiewicz et al. demonstrated that small PDA-based micelles $(\sim 10 \mathrm{~nm})$ with 2 kDa poly(ethylene glycol) (PEG) coating (PDA-PEG2000) could efficiently target tumors via EPR effect, enabling both in vivo tumor imaging and tumor growth inhibition. ${ }^{75}$ When loaded with a hydrophobic anticancer drug paclitaxel, PDA-PEG2000 micelles exhibited effective tumor accumulation and diffusion inside the tumor site, demonstrating great potential for theranostic applications.

In a very deliberate design, $\mathrm{Wu}$ and coworkers conjugated cytotoxic peptide magainin II (MGN-II) onto the surface of PDA micelles, via a fluorescence dye bridge (Fig. 7A). The welldefined PDA-based micelles were stable and highly cytotoxic to cancer cells. More interestingly, they exhibited enhanced 

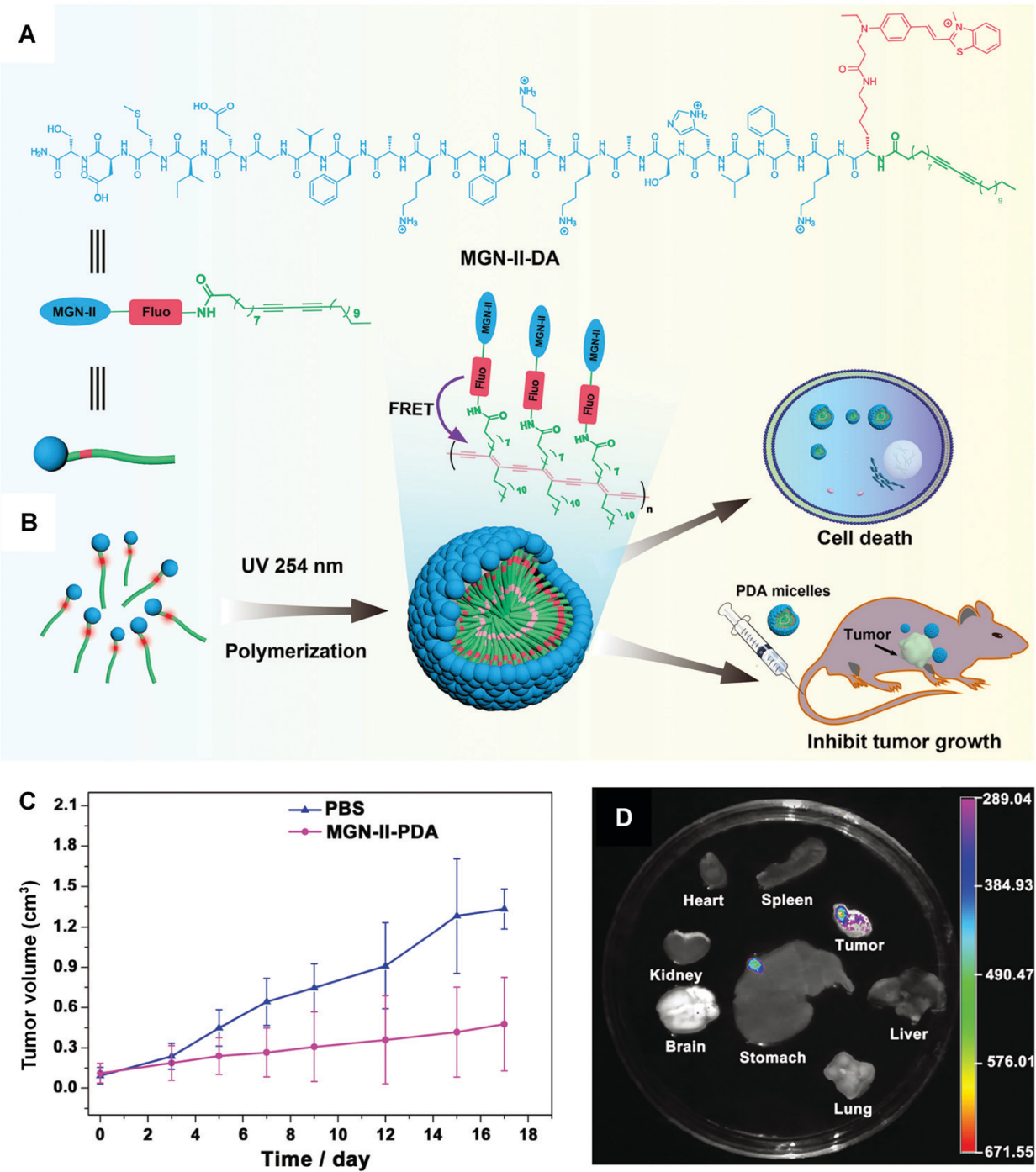

Fig. 7 (A) Scheme illustration of the structure of MGN-II-diacetylene. (B) Formation and applications of MGN-II-PDA micelles. (C) MGN-II-PDA blocks tumor growth in vivo. (D) The fluorescence images of excised mouse organs showing the targeting efficiency of MGN-II-PDA micelles. Adapted from ref. 76 with permission. Copyright 2014 The Royal Society of Chemistry.

fluorescence for live cell imaging, based on the energy transfer from the fluorescent dye to the backbone of PDA micelles. ${ }^{76}$ Moreover, PDA micelles could increase the local concentration of MGN-II and protect the peptide from degradation by increasing positive charges and hydrophobicity. ${ }^{77}$ The in vivo antitumor studies demonstrated that the modified PDA micelles possessed considerable anticancer effect with few side effects, offering a promising approach in the design of new micelles for cancer treatment.

\subsection{Other forms of PDA vectors}

In addition to liposomes and micelles, there are some other forms of PDA vectors with considerable capability in drug delivery. For instance, Zou and coworkers developed a PDA-based smart nanocontainer with photo and $\mathrm{pH}$ dual responses. By incorporating photo-responsive azobenzene derivative/cyclodextrin (Azo-CD) supramolecular complex into the $\mathrm{pH}$-responsive PDA vesicles matrix, they achieved regulated drug release by responding to weak acidic tumor microenvironment and remote photo irradiation. ${ }^{78}$ In 2017 , Somjid et al. reported a negatively charged PDA nanocapsules stabilized by photocleavable cationic gold nanoparticles to enable photoregulated release of hydrophobic drugs. ${ }^{79}$ The nanocapsules disintegrated upon UV light irradiation and released encapsulated model compound in consequence. The photoregulated release of the encapsulated drug was also irradiation time dependent, therefore providing a strategy for controlled drug release. Remy and coworkers reported a novel siRNA delivery system based on PDA nanofibers in $2018 .^{80}$ They investigated the silence efficiency of the siRNAassociated PDA nanofibers towards the targeted reporter gene in vitro. When administered in vivo, the PDA nanofibers could downregulate the targeted oncogene Lim-1 in nude mice efficiently. Guo and coworkers constructed programmable self-assembled PDA vesicles, which fluoresced only in the right sequential 
A

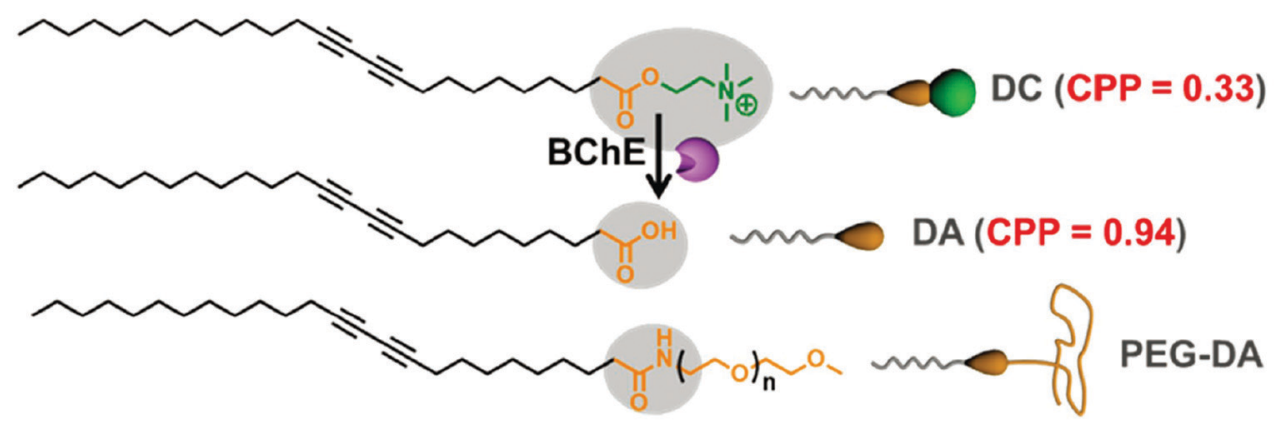

B

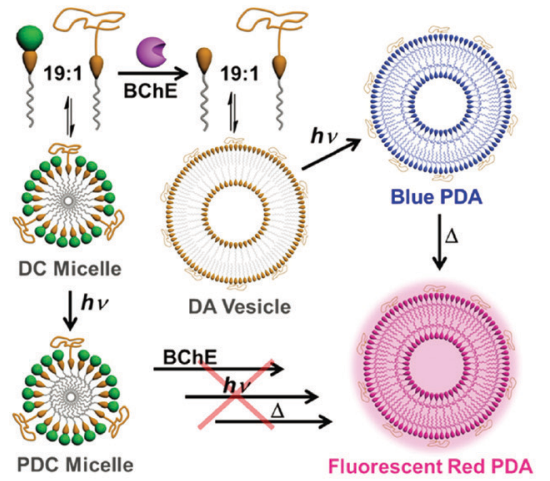

c

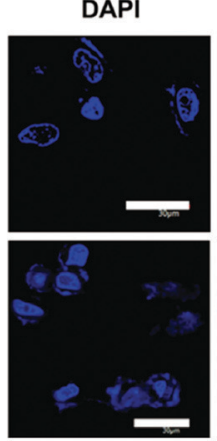

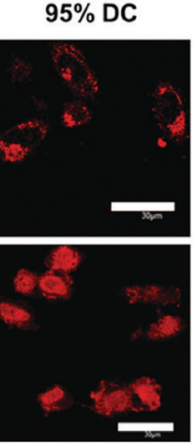

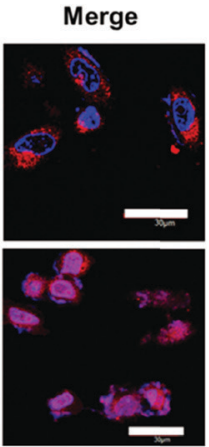

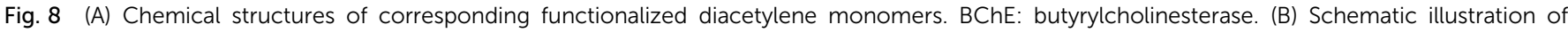

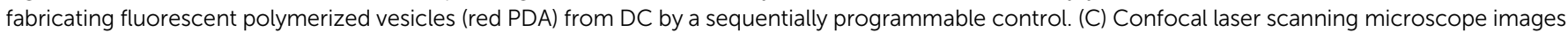

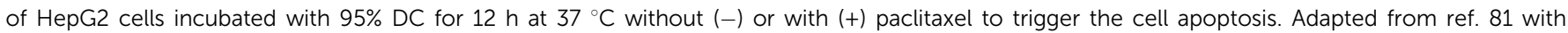
permission. Copyright 2017 Wiley-VCH Verlag GmbH \& Co. KGaA, Weinheim.

stimuli of enzyme, light, and heating inputs. ${ }^{81}$ Changing the order of stimuli would fail to result in fluorescent PDA vesicles. The PDA vesicles were applied to monitor the overexpressed cholinesterase in apoptotic cells, which represented a wash-free live-cell bioimaging approach with high signal-to-noise ratio (Fig. 8). Above all, the investigations of PDA materials for drug delivery have evidenced their highlights of nontoxicity, biocompatibility, and efficiency, demonstrating the potential of PDA-based smart materials for in vivo theranostic applications.

\section{Tissue engineering}

Tissue engineering aims to recreate some complex processes that a human embryo will undergo in its first eight weeks of life, by combining stem cells, scaffolds, and growth factors. ${ }^{82,83}$ When designing a scaffold for tissue engineering, there are several elements that need to take into consideration, such as biocompatibility with host tissues, tunable biodegradation rate, non-toxic degradation products, good mechanical strength, without or low immunogenicity, and suitable porosity for waste or nutrient transport and cell penetration. ${ }^{84-88}$ Polymers are one of the most widely used bioscaffold materials due to their good processing flexibility, biocompatibility, and biodegradability. However, natural and synthetic polymers both face difficulties in controlling their pore sizes, fiber diameters, and morphology. ${ }^{89}$ Peptide amphiphiles can self-assemble into supramolecular structures with controllable nano and microstructures, but their mechanical stability is poor due to unpolymerized amphiphilic peptides. Herein, topochemical diacetylene polymerization may be considered to stabilize the resulting selfassembly structure and avoid premature disassembly.

An early example of utilizing PDA materials in tissue engineering was reported by Tirrell and colleagues in $2006 .^{90}$ Because tri-peptide sequence arginine-glycine-aspartic acid (RGD) located in the extracellular matrix can promote cell adhesion, the authors synthesized a stable polymerized Langmuir-Blodgett film consisting of 10,12-tricosadiynoic acid (TDA) and celladhesion peptide glycine-arginine-glycine-aspartic acid-serineproline (GRGDSP). The strategy for preparing the target mixed polymerized monolayers was shown schematically in Fig. 9A. They investigated the effects of different peptide concentrations on cell adhesion, the results of which indicated that the films containing $10 \mathrm{~mol} \%$ TDA-GRGDSP showed the highest percentage of adherent cells (Fig. 9B). The optical microscope observation of the PDA films after a procedure of preliminary cell seeding, cell mechanical removal, and cell reseeding confirmed that the PDA films were recyclable for multiple reseeding of cells (Fig. 9C).

In 2009, Stupp and coworkers reported the application of PDA materials as a tissue engineering scaffold. ${ }^{91}$ Based on an integration of top-down techniques with bottom-up selfassembly, they have created an artificial matrix incorporating polymerizable acetylene groups in the hydrophobic segment of peptide amphiphiles (PAs). After the self-assembly of PA, the intra-fiber polymerization of acetylene monomers could produce 
A
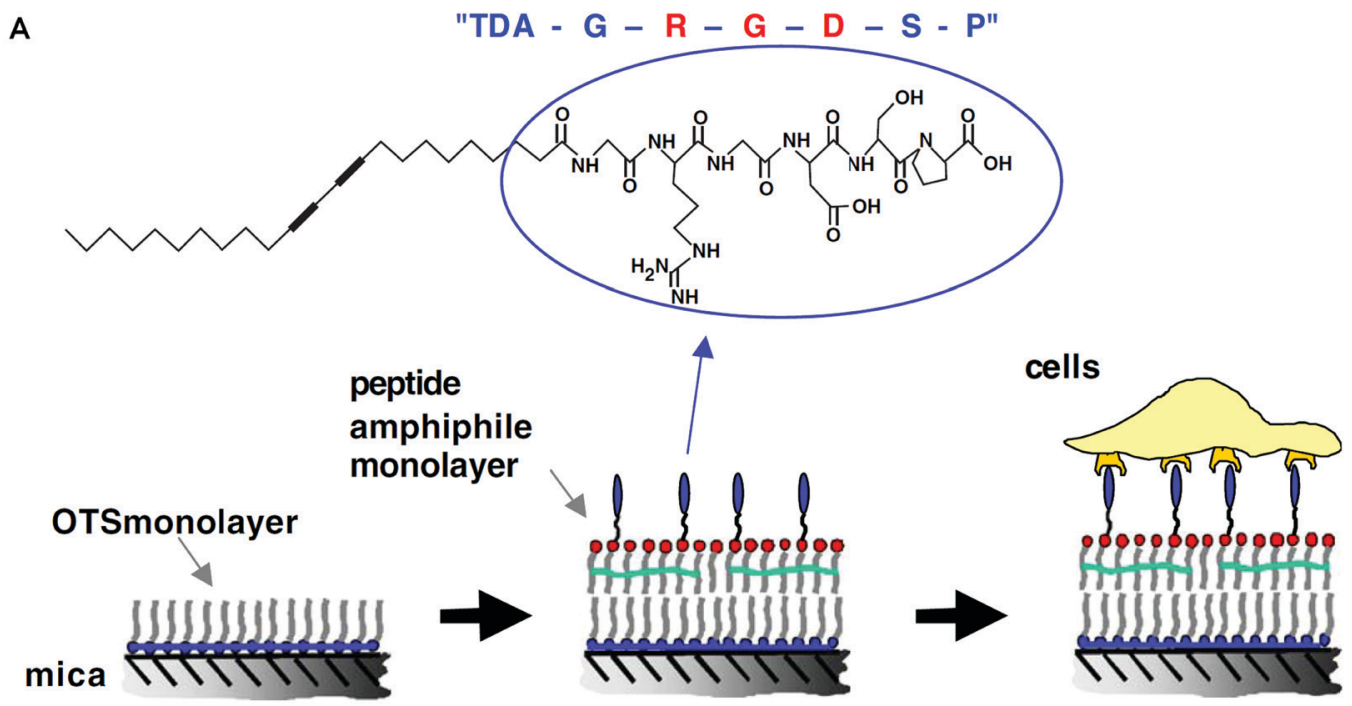

immobilized SAM on solid substrate (mica) polymerized peptideamphiphile on solid support cell attachment

B

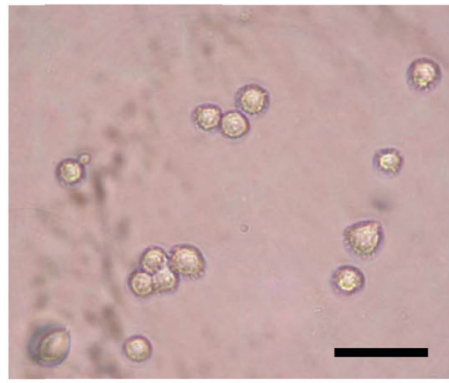

No peptide-amphiphile

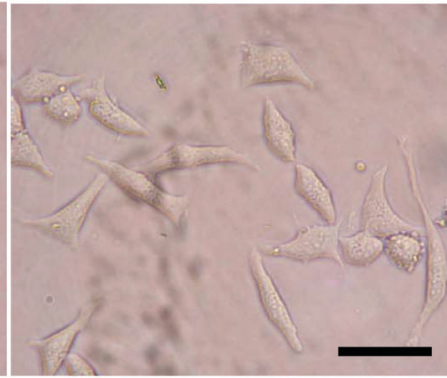

$10 \mathrm{~mol} \%$ peptide-amphiphile

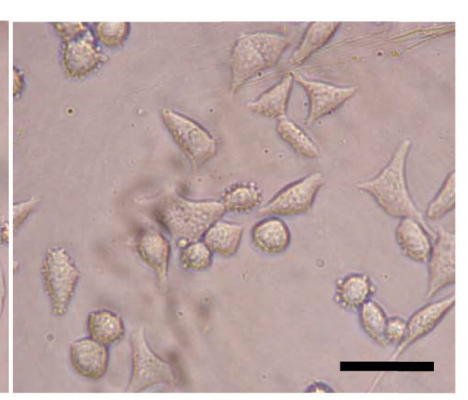

20 mol\% peptide-amphiphile

Fig. 9 (A) Schematic diagram of the strategy for constructing the biomimetic surfaces consisting of a supported polymerized peptide-amphiphile monolayer. (B) Optical micrographs of mouse fibroblast cells adhering to a supported polymerized peptide-amphiphile monolayer containing different amounts of TDA-GRGDSP. The scale bar is $50 \mu \mathrm{m}$. Adapted from ref. 90 with permission. Copyright 2005 Elsevier Ltd.

the morphological pattern with ordered architectures in both vertical and horizontal directions, forming the basis of a threedimensional matrix structure. They further compared the PA topographical patterns containing pre-aligned nanofibers with those containing randomly oriented nanofibers, in terms of their impacts on the phenotype of osteoblast differentiation of human mesenchymal stem cells (hMSC) as well as the competitive effects between nano- and microtextures on hMSC alignment. Their study indicated that hMSCs mainly aligned in the direction of the pre-aligned nanofibers even in the presence of hole microtextures, while osteoblastic differentiation was enhanced on hole microtextures in PA with randomly nanofibers.

Haridas et al. fabricated fibrous polymers for tissue engineering applications by self-assembly of lysine-appended PDAs. ${ }^{92}$ Instead of utilizing RGD adhesion peptides, they chose lysine (Lys) as an anchor unit since polylysine could support the adhesion of mammalian cells by electrostatic interaction between anionic sites on the cell membranes and cationic polylysine.$^{93}$ In this study, the appendant Lys with orthogonal protecting groups $t$-butyloxy carbonyl (Boc) and 9-fluorenylmethyloxycarbonyl (Fmoc) were introduced to adjust the chemical properties as well as wettability. AFM, TEM, and SEM confirmed the self-assembly abilities and the resulting fibrous morphology of the prepared monomers and polymers. They further assessed the stem cell attachment, spreading, and proliferation onto the prepared polymers. The results indicated that the hydrophilic polymer whose $\varepsilon$-amino groups were protected by Boc groups was more efficient to induce human dental pulp stem cells adhesion and osteogenic differentiation because of the porous and fibrous nature as well as the hydrophilicity of this polymer.

Komath and coworkers further investigated the dendritic lysine-appended PDA as a peptide matrix for periodontal regeneration in 2019. ${ }^{94}$ A diacetylene core appended with lysine was photopolymerized to form a polymeric dendritic lysine matrix (Lys-PDA). The authors fabricated an electrospun polycaprolactone (PCL) mat, which was then functionalized with Lys-PDA matrix (Fig. 10A). They investigated the cell adhesion, viability, and spreading on the Lys-PDA matrix surface in vitro. The alizarin red staining and Von Kossa staining of human 
A

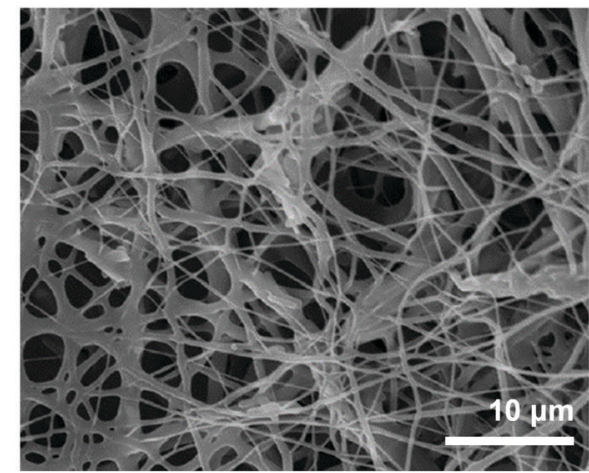

B
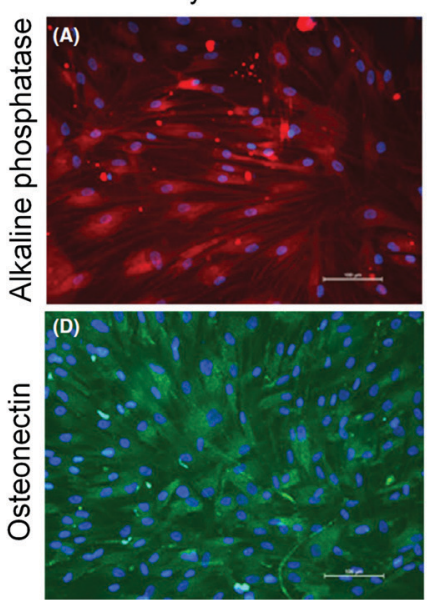

Positive control
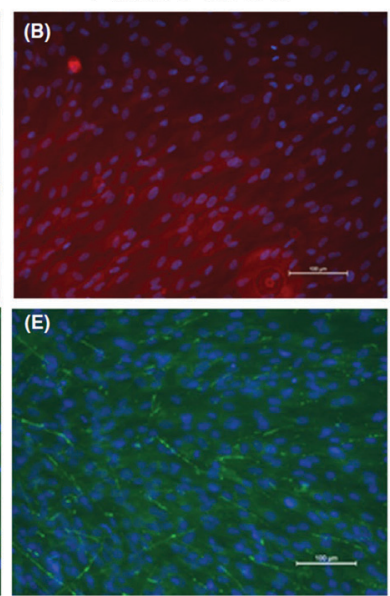

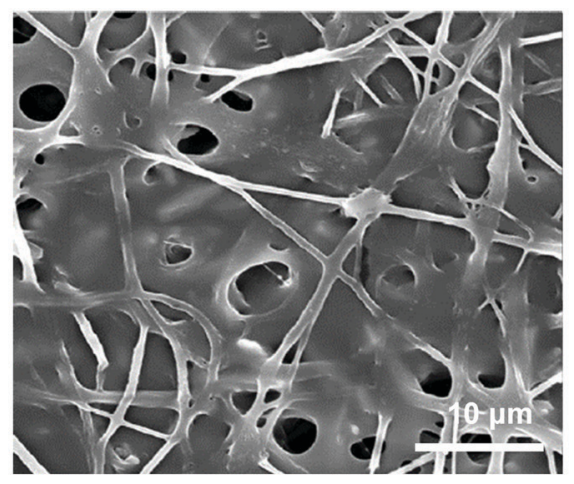

Negative control
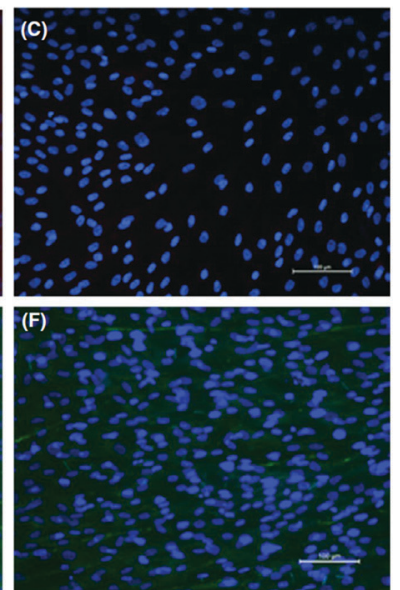

Fig. 10 (A) Scanning electronic microscope images of electrospun polycaprolactone (PCL) mat (left) and Lys-PDA bifunctionalized PCL mat (right). (B) Alkaline phosphatase enzyme and osteonectin expressions of hPDL cells on Lys-PDA matrix. Scale bar: $100 \mu \mathrm{m}$. Adapted from ref. 94 with permission. Copyright 2019 John Wiley \& Sons A/S.

periodontal ligament (hPDL) cells on Lys-PDA matrix indicated the early mineralization upon induction of osteogenic differentiation. They analyzed the presence of specific osteogenic markers such as alkaline phosphatase and osteonectin to further confirm osteogenic differentiation (Fig. 10B). The results of the study demonstrated that the Lys-PDA matrix gave rise to the possibility for diseased periodontal regeneration.

The application of PDA materials in the field of tissue engineering is an area that still needs more attention, given the many potential advantages PDA materials have. The addition of PDA materials greatly enhances the mechanical strength of the bioscaffold, and the unique optical properties of the PDA make it potential to detect signals indicating the cell adhesion and differentiation. The research on biodegradation of PDA is also beneficial to the field of bio-implantable materials in the future.

\section{Conclusion and outlook}

Since its first report by Wegner in $1969,{ }^{95}$ PDA has been extensively investigated in a wide range of optoelectronic and sensing applications. On the other hand, interest of utilizing
PDA-based smart materials in the biomedical area has emerged in recent decades. In this review, we focused on recent examples of designing PDA-based smart materials for biosensors, drug delivery, bioimaging, and tissue engineering. A few strategies for designing PDA-based biosensors have been summarized, including direct detection, indirect detection, sandwich method, and competitive binding method. We have also reviewed the PDA systems for drug delivery and bioimaging in the forms of liposomes, micelles, and beyond. Finally, several examples of PDA nanostructures for tissue engineering were discussed.

Although there has been substantial attention on the intriguing optical and mechanical properties of PDA-based biomaterials, the in vivo applications of these materials are still limited. PDA-based nanomaterials for drug delivery systems has unique features of stability, biocompatibility, and efficiency, which has endowed great advantages of applying these materials in living organisms. In addition, apart from the biomedical applications we reviewed above, the impact of other features of PDA on organisms is also worth investigating. For instance, we have developed a water-soluble PDA that showed ultrastrong Raman intensity of alkyne groups. The wavenumber of the alkyne Raman peak is right located in a region with no cellular background, making the material particular favorable 
as an bioorthogonal probe for targeted Raman imaging. ${ }^{96}$ Above all, PDA-based smart materials have demonstrated great potential for biomedical applications, and there are still plenty of undiscovered biological-related features of PDA waiting to be exploited.

\section{Conflicts of interest}

There are no conflicts of interest to declare.

\section{Acknowledgements}

This work is supported by the National Natural Science Foundation of China (21877042) and Shenzhen Science and Technology Project (JCYJ20170818161I36779).

\section{References}

1 H. Shirakawa, E. J. Louis, A. G. MacDiarmid, C. K. Chiang and A. J. Heeger, Synthesis of electrically conducting organic polymers: halogen derivatives of polyacetylene, $(\mathrm{CH})_{\mathrm{x}}, \mathrm{J}$. Chem. Soc., Chem. Commun., 1977, 16, 578-580.

2 D. T. McQuade, A. E. Pullen and T. M. Swager, Conjugated Polymer-Based Chemical Sensors, Chem. Rev., 2000, 100, 2537-2574.

3 H. N. Kim, Z. Guo, W. Zhu, J. Yoon and H. Tian, Recent progress on polymer-based fluorescent and colorimetric chemosensors, Chem. Soc. Rev., 2011, 40, 79-93.

4 S. Okada, S. Peng, W. Spevak and D. Charych, Color and Chromism of Polydiacetylene Vesicles, Acc. Chem. Res., 1998, 31, 229-239.

5 M. A. Reppy and B. A. Pindzola, Biosensing with polydiacetylene materials: structures, optical properties and applications, Chem. Commun., 2007, 4317-4338.

6 D. J. Ahn and J. M. Kim, Fluorogenic Polydiacetylene Supramolecules: Immobilization, Micropatterning, and Application to Label-Free Chemosensors, Acc. Chem. Res., 2008, 41, 805-816.

7 J. W. Lauher, F. W. Fowler and N. S. Goroff, Single-Crystalto-Single-Crystal Topochemical Polymerizations by Design, Acc. Chem. Res., 2008, 41, 1215-1229.

8 X. Sun, T. Chen, S. Huang, L. Li and H. Peng, Chromatic polydiacetylene with novel sensitivity, Chem. Soc. Rev., 2010, 39, 4244-4257.

9 X. Chen, G. Zhou, X. Peng and J. Yoon, Biosensors and chemosensors based on the optical responses of polydiacetylenes, Chem. Soc. Rev., 2012, 41, 4610-4630.

10 R. J. O. M. Hoofman, L. D. A. Siebbeles, M. P. de Haas, A. Hummel and D. Bloor, Anisotropy of the charge-carrier mobility in polydiacetylene crystals, J. Chem. Phys., 1998, 109, 1885-1893.

11 M. Akai-Kasaya, K. Shimizu, Y. Watanabe, A. Saito, M. Aono and Y. Kuwahara, Electronic structure of a polydiacetylene nanowire fabricated on highly ordered pyrolytic graphite, Phys. Rev. Lett., 2003, 91, 255501.
12 R. H. Baughman, Solid-state synthesis of large polymer single crystals, J. Polym. Sci., Polym. Phys. Ed., 1974, 12, 1511-1535.

13 M. D. Mowery, H. Menzel, M. Cai and C. E. Evans, Fabrication of Monolayers Containing Internal Molecular Scaffolding: Effect of Substrate Preparation, Langmuir, 1998, 14, 5594-5602.

14 R. W. Carpick, D. Y. Sasaki, M. S. Marcus, M. A. Eriksson and A. R. Burns, Polydiacetylene films: a review of recent investigations into chromogenic transitions and nanomechanical properties, J. Phys.: Condens. Matter, 2004, 16, R679-R697.

15 L. Luo, C. Wilhelm, A. Sun, C. P. Grey, J. W. Lauher and N. S. Goroff, Poly(diiododiacetylene): Preparation, Isolation, and Full Characterization of a Very Simple Poly(diacetylene), J. Am. Chem. Soc., 2008, 130, 7702-7709.

16 R. W. Carpick, D. Y. Sasaki and A. R. Burns, First Observation of Mechanochromism at the Nanometer Scale, Langmuir, 2000, 16, 1270-1278.

17 Y. Zhao, A. D. Slepkov, C. O. Akoto, R. McDonald, F. A. Hegmann and R. R. Tykwinski, Synthesis, structure, and nonlinear optical properties of cross-conjugated perphenylated iso-polydiacetylenes, Chemistry, 2004, 11, 321-329.

18 R. R. Chance, R. H. Baughman, H. Müller and C. J. Eckhardt, Thermochromism in a polydiacetylene crystal, J. Chem. Phys., 1977, 67, 3616-3618.

19 D. H. Charych, J. O. Nagy, W. Spevak and M. D. Bednarski, Direct colorimetric detection of a receptor-ligand interaction by a polymerized bilayer assembly, Science, 1993, 261, 585.

20 D. Charych, Q. Cheng, A. Reichert, G. Kuziemko, M. Stroh, J. O. Nagy, W. Spevak and R. C. Stevens, A 'litmus test' for molecular recognition using artificial membranes, Chem. Biol., 1996, 3, 113-120.

21 Q. Cheng and R. C. Stevens, Charge-Induced Chromatic Transition of Amino Acid-Derivatized Polydiacetylene Liposomes, Langmuir, 1998, 14, 1974-1976.

22 J. Song, Q. Cheng, S. Kopta and R. C. Stevens, Modulating Artificial Membrane Morphology: pH-Induced Chromatic Transition and Nanostructural Transformation of a Bolaamphiphilic Conjugated Polymer from Blue Helical Ribbons to Red Nanofibers, J. Am. Chem. Soc., 2001, 123, 3205-3213.

23 I. K. Kwon, M. S. Song, S. H. Won, S. P. Choi, M. Kim and S. J. Sim, Signal Amplification by Magnetic Force on Polydiacetylene Supramolecules for Detection of Prostate Cancer, Small, 2012, 8, 209-213.

24 Z. G. Soos, D. S. Galvao and S. Etemad, Fluorescence and excited-state structure of conjugated polymers, Adv. Mater., 1994, 6, 280-287.

25 J.-S. b. Filhol, J. R. M. Deschamps, S. G. Dutremez, B. Boury, T. Barisien, L. Legrand and M. Schott, Polymorphs and Colors of Polydiacetylenes: A First Principles Study, J. Am. Chem. Soc., 2009, 131, 6976-6988.

26 S. Lee, J.-Y. Kim, X. Chen and J. Yoon, Recent progress in stimuliinduced polydiacetylenes for sensing temperature, chemical and biological targets, Chem. Commun., 2016, 52, 9178-9196.

27 B. Yoon, S. Lee and J.-M. Kim, Recent conceptual and technological advances in polydiacetylene-based supramolecular chemosensors, Chem. Soc. Rev., 2009, 38, 1958-1968. 
28 E. Geiger, P. Hug and B. A. Keller, Chromatic Transitions in Polydiacetylene Langmuir-Blodgett Films due to Molecular Recognition at the Film Surface Studied by Spectroscopic Methods and Surface Analysis, Macromol. Chem. Phys., 2002, 203, 2422-2431.

29 B. Ma, Y. Fan, L. Zhang, X. Kong, Y. Li and J. Li, Direct colorimetric study on the interaction of Escherichia coli with mannose in polydiacetylene Langmuir-Blodgett films, Colloids Surf., B, 2003, 27, 209-213.

30 J. Lee, H.-J. Kim and J. Kim, Polydiacetylene Liposome Arrays for Selective Potassium Detection, J. Am. Chem. Soc., 2008, 130, 5010-5011.

31 J. Yoon, Y.-S. Jung and J.-M. Kim, A Combinatorial Approach for Colorimetric Differentiation of Organic Solvents Based on Conjugated Polymer-Embedded Electrospun Fibers, Adv. Funct. Mater., 2009, 19, 209-214.

32 B. Yoon, H. Shin, E.-M. Kang, D. W. Cho, K. Shin, H. Chung, C. W. Lee and J.-M. Kim, Inkjet-Compatible Single-Component Polydiacetylene Precursors for Thermochromic Paper Sensors, ACS Appl. Mater. Interfaces, 2013, 5, 4527-4535.

33 J. T. Wen, K. Bohorquez and H. Tsutsui, Polydiacetylenecoated polyvinylidene fluoride strip aptasensor for colorimetric detection of zinc(II), Sens. Actuators, B, 2016, 232, 313-317.

34 F. Jannah, J.-H. Kim, J.-W. Lee, J.-M. Kim, J.-M. Kim and H. Lee, Immobilized Polydiacetylene Lipid Vesicles on Polydimethylsiloxane Micropillars as a Surfactin-Based Label-Free Bacterial Sensor Platform, Front. Mater., 2018, $5,57$.

35 F. Hassan, C. Gentry-Weeks, M. Reynolds and Y. V. Li, Study on microstructure and mechanical properties of polydiacetylene composite biosensors, J. Appl. Polym. Sci., 2019, 136, 47877.

36 F. Jannah and J.-M. Kim, pH-sensitive colorimetric polydiacetylene vesicles for urease sensing, Dyes Pigm., 2019, 169, 15-21.

37 C. Kim and K. Lee, Polydiacetylene (PDA) Liposome-Based Immunosensor for the Detection of Exosomes, Biomacromolecules, 2019, 20, 3392-3398.

38 S. U. Son, S. B. Seo, S. Jang, J. Choi, J.-W. Lim, D. K. Lee, H. Kim, S. Seo, T. Kang, J. Jung and E.-K. Lim, Naked-eye detection of pandemic influenza a (pH1N1) virus by polydiacetylene (PDA)-based paper sensor as a point-of-care diagnostic platform, Sens. Actuators, B, 2019, 291, 257-265.

39 M.-K. Park, K.-W. Kim, D. J. Ahn and M.-K. Oh, Label-free detection of bacterial RNA using polydiacetylene-based biochip, Biosens. Bioelectron., 2012, 35, 44-49.

40 S. Song, K. Ha, K. Guk, S.-G. Hwang, J. M. Choi, T. Kang, P. Bae, J. Jung and E.-K. Lim, Colorimetric detection of influenza A (H1N1) virus by a peptide-functionalized polydiacetylene (PEP-PDA) nanosensor, RSC Adv., 2016, 6, 48566-48570.

41 Z. Ma, J. Li, M. Liu, J. Cao, Z. Zou, J. Tu and L. Jiang, Colorimetric Detection of Escherichia coli by Polydiacetylene Vesicles Functionalized with Glycolipid, J. Am. Chem. Soc., 1998, 120, 12678-12679.
42 Q. Cheng and R. C. Stevens, Coupling of an induced fit enzyme to polydiacetylene thin films: Colorimetric detection of glucose, Adv. Mater., 1997, 9, 481-483.

43 Y. K. Jung, T. W. Kim, H. G. Park and H. T. Soh, Specific Colorimetric Detection of Proteins Using Bidentate AptamerConjugated Polydiacetylene (PDA) Liposomes, Adv. Funct. Mater., 2010, 20, 3092-3097.

44 J. Deng, Z. Sheng, K. Zhou, M. Duan, C.-Y. Yu and L. Jiang, Construction of effective receptor for recognition of Avian Influenza $\mathrm{H} 5 \mathrm{~N} 1$ protein HA1 by assembly of monohead glycolipids on polydiacetylene vesicle surface, Bioconjugate Chem., 2009, 20, 533-537.

45 C. H. Park, J. P. Kim, S. W. Lee, N. L. Jeon, P. J. Yoo and S. J. Sim, A direct, multiplex biosensor platform for pathogen detection based on cross-linked polydiacetylene (PDA) supramolecules, Adv. Funct. Mater., 2009, 19, 3703-3710.

46 Y. K. Jung and H. G. Park, Colorimetric detection of clinical DNA samples using an intercalator-conjugated polydiacetylene sensor, Biosens. Bioelectron., 2015, 72, 127-132.

47 C. Medhi, J. B. O. Mitchell, S. L. Price and A. B. Tabor, Electrostatic factors in DNA intercalation, Biopolymers, 1999, 52, 84-93.

48 H. Otsuka, E. Uchimura, H. Koshino, T. Okano and K. Kataoka, Anomalous binding profile of phenylboronic acid with $n$-acetylneuraminic acid (Neu5Ac) in aqueous solution with varying pH, J. Am. Chem. Soc., 2003, 125, 3493-3502.

49 A. Matsumoto, N. Sato, K. Kataoka and Y. Miyahara, Noninvasive sialic acid detection at cell membrane by using phenylboronic acid modified self-assembled monolayer gold electrode, J. Am. Chem. Soc., 2009, 131, 12022-12023.

50 E. Han, L. Ding, R. Qian, L. Bao and H. Ju, Sensitive chemiluminescent imaging for chemoselective analysis of glycan expression on living cells using a multifunctional nanoprobe, Anal. Chem., 2012, 84, 1452-1458.

51 D. E. Wang, J. Yan, J. Jiang, X. Liu, C. Tian, J. Xu, M. S. Yuan, X. Han and J. Wang, Polydiacetylene liposomes with phenylboronic acid tags: a fluorescence turn-on sensor for sialic acid detection and cell-surface glycan imaging, Nanoscale, 2018, 10, 4570-4578.

52 J. Park, S. K. Ku, D. Seo, K. Hur, H. Jeon, D. Shvartsman, H.-K. Seok, D. J. Mooney and K. Lee, Label-free bacterial detection using polydiacetylene liposomes, Chem. Commun., 2016, 52, 10346-10349.

53 C. Y. Wang, Synthesis and crystal structures of cobalt(III) and zinc(II) complexes derived from 4-chloro-2-[(2-morpholin-4ylethylimino)methyl]phenol with urease inhibitory activity, Russ. J. Coord. Chem., 2010, 36, 177-182.

54 D.-E. Wang, X. Gao, G. Li, T. Xue, H. Yang and H. Xu, Facile colorimetric assay of alkaline phosphatase activity using polydiacetylene liposomes with calcium ions and pyrophosphate, Sens. Actuators, B, 2019, 289, 85-92.

55 M. P. Whyte, Physiological role of alkaline phosphatase explored in hypophosphatasia, Ann. N. Y. Acad. Sci., 2010, 1192, 190-200.

56 I. K. Kwon, J. P. Kim and S. J. Sim, Enhancement of sensitivity using hybrid stimulus for the diagnosis of 
prostate cancer based on polydiacetylene (PDA) supramolecules, Biosens. Bioelectron., 2010, 26, 1548-1553.

57 J. P. Kim, I. K. Kwon and S. J. Sim, The strategy of signal amplification for ultrasensitive detection of hIgE based on aptamer-modified poly(di-acetylene) supramolecules, Biosens. Bioelectron., 2011, 26, 4823-4827.

58 J. U. Lee, J. H. Jeong, D. S. Lee and S. J. Sim, Signal enhancement strategy for a micro-arrayed polydiacetylene (PDA) immunosensor using enzyme-catalyzed precipitation, Biosens. Bioelectron., 2014, 61, 314-320.

59 J. Brockgreitens, S. Ahmed and A. Abbas, Kinetic analysis of $\alpha$-cyclodextrin interactions using polydiacetylene liposomes, J. Inclusion Phenom. Macrocyclic Chem., 2014, 81, 423-427.

60 Y. Zhu, D. Qiu, G. Yang, M. Wang, Q. Zhang, P. Wang, H. Ming, D. Zhang, Y. Yu, G. Zou, R. Badugu and J. R. Lakowicz, Selective and sensitive detection of MiRNA-21 based on gold-nanorod functionalized polydiacetylene microtube waveguide, Biosens. Bioelectron., 2016, 85, 198-204.

61 S. Dromi, V. Frenkel, A. Luk, B. Traughber, M. Angstadt, M. Bur, J. Poff, J. Xie, S. K. Libutti, K. C. P. Li and B. J. Wood, Pulsed-high intensity focused ultrasound and low temperature-sensitive liposomes for enhanced targeted drug delivery and antitumor effect, Clin. Cancer Res., 2007, 13, 2722.

62 U. Bulbake, S. Doppalapudi, N. Kommineni and W. Khan, Liposomal formulations in clinical use: An updated review, Pharmaceutics, 2017, 9, 12.

63 G. Qin, Z. Li, R. Xia, F. Li, B. E. O’Neill, J. T. Goodwin, H. A. Khant, W. Chiu and K. C. Li, Partially polymerized liposomes: stable against leakage yet capable of instantaneous release for remote controlled drug delivery, Nanotechnology, 2011, 22, 155605.

64 L. A. Porter, D. Ji, S. L. Westcott, M. Graupe, R. S. Czernuszewicz, N. J. Halas and T. R. Lee, Gold and Silver Nanoparticles functionalized by the adsorption of dialkyl disulfides, Langmuir, 1998, 14, 7378-7386.

65 H. Maeda, Macromolecular therapeutics in cancer treatment: The EPR effect and beyond, J. Controlled Release, 2012, 164, 138-144.

66 H. Maeda, H. Nakamura and J. Fang, The EPR effect for macromolecular drug delivery to solid tumors: Improvement of tumor uptake, lowering of systemic toxicity, and distinct tumor imaging in vivo, Adv. Drug Delivery Rev., 2013, 65, 71-79.

67 J. S. Abrams, S. E. Howe, N. Becerra, P. Kohli and V. Konjufca, Immunogenicity of antigen-conjugated biodegradable polydiacetylene liposomes administered mucosally, J. Biomed. Mater. Res., Part A, 2017, 105, 557-565.

68 J. Nicolas, S. Mura, D. Brambilla, N. Mackiewicz and P. Couvreur, Design, functionalization strategies and biomedical applications of targeted biodegradable/biocompatible polymer-based nanocarriers for drug delivery, Chem. Soc. Rev., 2013, 42, 1147-1235.

69 L. Li, X. An and X. Yan, Folate-polydiacetylene-liposome for tumor targeted drug delivery and fluorescent tracing, Colloids Surf., B, 2015, 134, 235-239.
70 E. Gravel, J. Ogier, T. Arnauld, N. Mackiewicz, F. Ducongé and E. Doris, Drug delivery and imaging with polydiacetylene micelles, Chem. - Eur. J., 2012, 18, 400-408.

71 I. Theodorou, P. Anilkumar, B. Lelandais, D. Clarisse, A. Doerflinger, E. Gravel, F. Ducongé and E. Doris, Stable and compact zwitterionic polydiacetylene micelles with tumor-targeting properties, Chem. Commun., 2015, 51, 14937-14940.

72 A. Doerflinger, N. N. Quang, E. Gravel, G. Pinna, M. Vandamme, F. Ducongé and E. Doris, Biotin-functionalized targeted polydiacetylene micelles, Chem. Commun., 2018, 54, 3613-3616.

73 A. Doerflinger, N. N. Quang, E. Gravel, F. Ducongé and E. Doris, Aptamer-decorated polydiacetylene micelles with improved of cancer cells, Int. J. Pharm., 2019, 565, 59-63.

74 E. Romero-Ben, T. Mena Barragán, E. García de Dionisio, E. M. Sánchez-Fernández, J. M. Garcia Fernández, E. GuillénMancina, M. López-Lázaro and N. Khiar, Mannose-coated polydiacetylene (PDA)-based nanomicelles: synthesis, interaction with concanavalin $\mathrm{A}$ and application in the water solubilization and delivery of hydrophobic molecules, J. Mater. Chem. B, 2019, 7, 5930-5946.

75 N. Mackiewicz, E. Gravel, A. Garofalakis, J. Ogier, J. John, D. M. Dupont, K. Gombert, B. Tavitian, E. Doris and F. Ducongé, Tumor-targeted polydiacetylene micelles for in vivo imaging and drug delivery, Small, 2011, 7, 2786-2792.

76 D. Yang, R. Zou, Y. Zhu, B. Liu, D. Yao, J. Jiang, J. Wu and H. Tian, Magainin II modified polydiacetylene micelles for cancer therapy, Nanoscale, 2014, 6, 14772-14783.

77 K. C. Lee, P. A. Carlson, A. S. Goldstein, P. Yager and M. H. Gelb, Protection of a decapeptide from proteolytic cleavage by lipidation and self-assembly into high-axialratio microstructures: a kinetic and structural study, Langmuir, 1999, 15, 5500-5508.

78 J. Li, Z. Yu, H. Jiang, G. Zou and Q. Zhang, Photo and $\mathrm{pH}$ dual-responsive polydiacetylene smart nanocontainer, Mater. Chem. Phys., 2012, 136, 219-224.

79 S. Somjid, A. Chompoosor, S. Kanokmedhakul and S. Teerasong, Photoregulation of gold nanoparticles stabilized in a diacetylenic nanocapsule, J. Nanomater., 2017, 1-6.

80 P. Neuberg, I. Hamaidi, S. Danilin, M. Ripoll, V. Lindner, M. Nothisen, A. Wagner, A. Kichler, T. Massfelder and J.-S. Remy, Polydiacetylenic nanofibers as new siRNA vehicles for in vitro and in vivo delivery, Nanoscale, 2018, 10, 1587-1590.

81 S. Peng, Y.-C. Pan, Y. Wang, Z. Xu, C. Chen, D. Ding, Y. Wang and D.-S. Guo, Sequentially programmable and cellularly selective assembly of fluorescent polymerized vesicles for monitoring cell apoptosis, Adv. Sci., 2017, 4, 1700310.

82 E. S. Place, N. D. Evans and M. M. Stevens, Complexity in biomaterials for tissue engineering, Nat. Mater., 2009, 8, 457-470.

83 S. Varun, T. Saket, B. Lianne and S. K. Wasim, The role of tissue engineering in achilles tendon repair: a review, Curr. Stem Cell Res. Ther., 2015, 10, 31-36.

84 S. P. Zustiak and J. B. Leach, Hydrolytically degradable poly(ethylene glycol) hydrogel scaffolds with tunable 
degradation and mechanical properties, Biomacromolecules, 2010, 11, 1348-1357.

85 S. Van Vlierberghe, P. Dubruel and E. Schacht, Biopolymerbased hydrogels as scaffolds for tissue engineering applications: A Review, Biomacromolecules, 2011, 12, 1387-1408.

86 B. Guo and P. X. Ma, Synthetic biodegradable functional polymers for tissue engineering: a brief review, Sci. China: Chem., 2014, 57, 490-500.

87 Y. Wu, L. Wang, X. Zhao, S. Hou, B. Guo and P. X. Ma, Selfhealing supramolecular bioelastomers with shape memory property as a multifunctional platform for biomedical applications via modular assembly, Biomaterials, 2016, 104, 18-31.

88 B. Guo and P. X. Ma, Conducting Polymers for Tissue Engineering, Biomacromolecules, 2018, 19, 1764-1782.

89 L. A. Smith and P. X. Ma, Nano-fibrous scaffolds for tissue engineering, Colloids Surf., B, 2004, 39, 125-131.

90 M. A. Biesalski, A. Knaebel, R. Tu and M. Tirrell, Cell adhesion on a polymerized peptide-amphiphile monolayer, Biomaterials, 2006, 27, 1259-1269.

91 A. Mata, L. Hsu, R. Capito, C. Aparicio, K. Henrikson and S. I. Stupp, Micropatterning of bioactive self-assembling gels, Soft Matter, 2009, 5, 1228-1236.
92 V. Haridas, S. Sadanandan, P. Y. Collart-Dutilleul, S. Gronthos and N. H. Voelcker, Lysine-appended polydiacetylene scaffolds for human mesenchymal stem cells, Biomacromolecules, 2014, 15, 582-590.

93 S. K. Samal, M. Dash, S. Van Vlierberghe, D. L. Kaplan, E. Chiellini, C. van Blitterswijk, L. Moroni and P. Dubruel, Cationic polymers and their therapeutic potential, Chem. Soc. Rev., 2012, 41, 7147-7194.

94 E. C. Das, S. Dhawan, J. Babu, P. R. Anil Kumar, T. V. Kumary, V. Haridas and M. Komath, Self-assembling polymeric dendritic peptide as functional osteogenic matrix for periodontal regeneration scaffolds-an in vitro study, J. Periodontal Res., 2019, 54, 468-480.

95 G. Wegner, Topochemische Reaktionen von Monomeren mit konjugierten Dreifachbindungen/Topochemical reactions of monomers with conjugated triple Bonds, Z. Naturforsch., B: Anorg. Chem., Org. Chem., Biochem., Biophys., Biol., 1969, 24, 824-832.

96 S. Tian, H. Li, Z. Li, H. T. Tang, M. Yin, Y. Chen, S. Wang, Y. Gao, X. Yang, F. Meng, J. W. Lauher, P. Wang and L. Luo, Polydiacetylene-based ultrastrong bioorthogonal Raman probes for targeted live-cell Raman imaging, Nat. Commun., 2020, 11, 81. 